\title{
A Comparative Study on Social Media Addiction of High School and University Students
}

\author{
Ali Simsek \\ Anadolu University, Turkey \\ ORCID: 0000-0002-0960-6805 \\ Kemal Elciyar \\ Anadolu University, Turkey \\ ORCID: 0000-0002-7820-2978 \\ Taner Kizilhan \\ Anadolu University, Turkey \\ ORCID: 0000-0002-3501-415X
}

\begin{abstract}
Considering that social media addiction is probably the most recent type of technology addiction, the present study was designed based on the six components suggested by Griffiths (2013). Toward the main purpose of the study, the "Bergen Facebook Addiction Scale" was adapted to social media addiction and translated into Turkish. After the validation process, it was administered to a total of 700 students; of them 397 were high school students and 303 were university students. The data collection instrument included 18 five-point Likert-type items in six categories, along with 5 structured items regarding demographics of the respondents. In addition to the original findings of the present study, similar research on social media addiction in some other countries were examined for comparisons. The results showed that both university students and high school students have a moderate level of addiction to social media. Being a university or high school student does not make any difference on the level of social media addiction. However, significant differences were found regarding gender, duration of use, department at the university, and type of high school. Finally, the results of the study show certain similarities and a few differences with the results of the studies conducted in other countries.
\end{abstract}

Keywords: Social media; Internet addiction; Social media addiction; University students; High school students

\section{Introduction}

Traditionally, the concept of addiction is based on a medical model, and it expresses extreme physical or psychological desire and commitment to a physical object. However, researchers argue that addiction should be addressed to cover a broader behavioral domain (Shaffer, 1996, p.462) including social and psychological behaviors. In this context, Griffiths (2013) proposed the concept of technology addiction. He has defined technology addiction as the actions which are non-chemical but behavioral, and involve extreme human-machine interaction. Also, technology 
addiction is portrayed as a passive behavior like watching TV, playing a video game, and chatting online.

Internet addiction is a special type of technology addiction. The term "Internet addiction" was defined for the first time by Ivan Goldberg in 1995 as a psychological illness (cited in Kim, 2009). Different conceptualizations were used for abusing Internet. Young (1998) named it as "internet addiction", Morahan-Martin and Schumacher (2000) used the term "pathological internet use", Davis, Flett and Besser (2002) labelled it as "problematic internet use" (cited in Caplan, 2002, p.554).

Young (1998) suggests five types of Internet addiction: (a) cyber sexual addiction, (b) cyber relationship addiction to online friendships or affairs, (c) net compulsions to online gambling, auctions or obsessive trading, (d) information overload to compulsive web surfing or databases searches, and (e) computer addiction to game playing or programming.

When properly used, Internet is an important technology that provides people with vital skills for the 21st century such as information access, problem solving, and self-directed learning. However, when Internet is used unconsciously, it can cause anxiety or fear and negatively affect personal development (Colwell \& Kato, 2003; Kerberg, 2005). In addition, excessive use of Internet may have detrimental effects on biological, physiological, psychological and social development of the user (Caplan, 2002). In this context, Internet addiction has gradually become a serious problem. Thus, although it was not mentioned in the previous editions, American Psychiatric Association (APA) has added Internet addiction, with a particular reference to online gambling, as a mental illness to the fifth edition of the Handbook of Diagnostic and Statistical Manual of Mental Disorders (American Psychiatric Association, 2013).

In many areas of behavioral addiction, it has been debated that whether some extreme behaviors can really be regarded as addiction or not. Social media addiction, as a special type of Internet addiction, has been discussed in this context. Griffiths (2013) made an important contribution to this discussion by suggesting six essential components to describe a behavior as addiction. These six components are salience, tolerance, mood modification, relapse, withdrawal, and conflict (Griffiths, 2013, p.121). He states that a behavior can be defined as addiction if it has these six components. Then, the critical question becomes what does each of these six components mean? To make the subject or issue more understandable, Griffiths (2013) explains these six components as follows:

- Salience: This occurs when social networking becomes the single most important activity in a person's life and dominates his or her thinking, feelings, and behavior. For instance, even if people are not actually engaged in social networking, they will be constantly thinking about the next time that they will be.

- Mood modification: This refers to the subjective experiences that people report as a consequence of social networking and can be seen as a coping strategy (i.e., they experience an arousing "buzz" or a "high" or, paradoxically, a tranquilizing feeling of "escape" or "numbing").

- Tolerance: This is the process whereby increasing amounts of social networking activity are required to achieve the former mood-modifying effects. This basically means that for people engaged in social networking, they gradually build up the amount of the time they spend social networking every day. 
- Withdrawal symptoms: These are the unpleasant feeling states and/or physical effects (e.g., the shakes, moodiness, irritability) that occur when people are unable to engage in social networking because they are ill, on vacation, prohibited etc.

- Conflict: This refers to the conflicts between a person and those around that person (interpersonal), conflicts with other activities (social life, hobbies, and interests), or from within the individual himself or herself (intrapsychic conflict and/or subjective feelings of loss of control) that are concerned with spending too much time on social networking.

- Relapse: This is the tendency or desire for repeated reversions to earlier patterns of excessive social networking to recur and for even the most extreme patterns typical of the height of excessive social networking to be quickly restored after periods of personal control.

Around the world, a number of studies have been conducted on whether the Internet and its applications are addictive or not. For example, in a study conducted in China which was attended by 2,620 students, it was identified that $24 \%$ of the participants were addicted to social media. One of the notable points here is that students who have developed high scores on addiction have got low scores on time management. In addition, internet-addicted adolescents provided meaningful results in emotional symptoms, problem management, hyperactivity situations and lower social behavior scores (Cao et al, 2007).

Social network sites are virtual communities where users can create individual or public profiles, interact with friends in real life, and come together with other people based on common interests (Kuss \& Griffiths, 2011, p.3529). When considering the history of social networking sites, the first social networking example - Six Degrees - was published in 1997 on a basis that would allow everyone to connect via six-degree distance. In 2004, Facebook was established, which is now considered as the most successful social networking site. The site has expanded very quickly and has become a global network with more than 2.3 billion users as of January 2019 (wearesocial.org), with more than half of the users logging every day. Social networks are predominantly used for social purposes and for the continuation as well as the development of individuals' offline connections (Kuss \& Griffiths, 2011, p.3528).

Nowadays, intensive use of social media applications by ordinary users has increased; and this has raised the concerns for addiction. Surveys consistently show that young people and students benefit most from social networks in the general population (Kuss \& Griffiths, 2011). The rapid increase in the use of online networks -mainly on the basis of time spent- has led some researchers to argue that excessive social networking can lead to addiction in individuals (Griffiths \& Pontes, 2014, p.120; Sussman et al., 2011). From time to time, individuals can have a variety of actions that are potentially addictive, such as using social networks extensively. These behaviors do not always have to be addictive, sometimes they are just behaviors which lead to addiction.

Studies on social media addiction levels have produced inconclusive results because some are concerned about negative consequences of intensive use, while others have focused on gains through the use of social media. However, the latest research showed that greater access to social media brings greater concerns. For many institutions, social media addiction has become a major concern since the beginning years of social networks. For example, Davies and Cranston (2008) found that participants (120 managers and practitioners working on youth) were afraid that online social networking would replace other activities and face-to-face social interaction 
among people. When participants were asked to identify the risks associated with online social networks, $23 \%$ reported addiction as a source of concern and the others reported exposure to bullying (53\%), disclosure of personal information (35\%), and sexual assault (22\%).

Koc and Gulyagci (2013) found that Facebook addiction was related to factors such as usage time, social motivations, depression, anxiety, and insomnia. However, they did not find any significant relationships regarding demographic factors. In several studies, researchers found that frequent access to Facebook is associated with clinical manifestations of psychiatric disorders (Rosen et al., 2013), the excessive use of social media can disrupt interpersonal relationships by jeopardizing the quality of relationships people develop with others (Tokunaga, 2011), and it might reduce self-esteem (Peter \& Valkenburg, 2006).

There are a number of social and psychological factors that affect social media addiction. The role of parents is particularly emphasized by researchers. It has been reported that while the lack of family love (Huang \& Leung, 2009) and the conflict between parents and children (Yen, Ko, \& Yen, 2007) may lead to dependent behaviors; supportive parental supervision (Lin, Ko, \& $\mathrm{Wu}, 2009)$ decreases the impact on dependent behaviors. Many other variables, such as the need to establish a close relationship, narcissistic behavior, excess of leisure time, and the limitations of the social environment may be determinants of social media addiction. At the same time, negative effects on adolescents, who are practically students, can sometimes be reflected in academic performance and social capital.

In short, social media addiction is an ever-increasing problem in the 21st century. For this reason, a number of studies were conducted in various countries on this subject. Each study presents a new outcome, explains reasons and effects of the social media addiction, and presents new ways of solutions. However, the problem is getting bigger and becoming more prevalent every day and public authorities, educators, and parents know neither the scope nor the extent of this challenge. Turkey is no exception as a country that ranks among the top countries in the world when it comes to the use of social media. Considering that the possible actions against addictive uses of social networking sites depend largely on understanding the seriousness of the problem, this study may shed some light on the current situation among particularly young users in Turkey. This rationale sets the need for the current study.

Within this framework, the main purpose of the present study is to identify the social media addiction level of high school and university students based on internationally-recognized measures and compare it with the situation in other countries where similar instrumentation was used. Toward this main purpose, the following research questions were investigated:

- What is the social media addiction levels of high school and university students based on internationally-recognized measures?

- Are there significant differences between social media addiction levels of high school and university students in general and in terms of sub-categories of technology addiction?

- Are there significant differences in social media addiction levels of students in terms of gender, school type, department, and duration of daily use?

- Is social media addiction level of Turkish students different from the social media addiction levels of students in other countries? 


\section{Methods}

\section{Participants}

The literature suggests that majority of social media users are teenagers and young adults. In practical terms, high school students are teenagers and university students are young adults. Considering that most of the social media users fall within these two categories, their age groups make them potentially more vulnerable for social media addiction compared to other segments of the population. For this reason, the sample of the present study was chosen from among high school and university students. Table 1 shows the composition of the sample.

Table 1. Characteristics of the Participants

\begin{tabular}{lrrrr}
\hline & Female & Male & Total \\
\cline { 3 - 4 } High School & 205 & 192 & 397 \\
Anatolian High School & 89 & 102 & 191 \\
Science High School & 116 & 90 & 206 \\
University & 140 & 163 & 303 \\
Department of Communication Design & 79 & 87 & 166 \\
Department of Journalism & 12 & 20 & 32 \\
Department of Cinema-TV & 12 & 19 & 31 \\
Department of Public Relations & 37 & 37 & 74 \\
Total & 345 & 355 & 700 \\
\hline
\end{tabular}

The sample of the study consisted of 700 students. Of them, 345 (49\%) were female and 355 (51\%) were male. Gender distribution was almost equal. As far as their schools are concerned, 397 (57\%) of the participants were high school students and 303 (43\%) participants were university students. High schools students formed the majority with a difference of $14 \%$. Regarding the amount of time they spend on social media per day, $115(16 \%)$ students used social media for less than an hour, 344 (49\%) used for 2-3 hours, 145 (21\%) used for 4-5 hours, and 96 (14\%) used for more than 5 hours. It appears that approximately $85 \%$ of students use social media for more than 2 hours per day. The students in the sample also had a high level of mobile phone ownership (96\%).

The composition of the high school sub-sample is generally similar to that of the total sample, partly due to the fact that it formed the majority. Within the high school sub-sample, $52 \%$ were female and $48 \%$ were male; $48 \%$ were in Anatolian High School and 52\% were in Science High School; $84 \%$ use social media for more than 2 hours a day; and $96 \%$ have their own mobile phone.

The composition of the university sub-sample is a little bit different. Within this group, $46 \%$ were female and 54\% were male; $55 \%$ were in the Department of Communication Design and Management, $11 \%$ were in the Department of Journalism, 10\% were in the Department of Cinema and Television, 24\% were in the Department of Public Relations and Advertising; $87 \%$ use social media for more than 2 hours a day; and 99\% owned a mobile phone. 
General demographics of the total sample as well as the sub-samples are in compliance with the selection criteria employed in the study; that is, the researchers have assumed that both the students in the private high school system and the university students in the Faculty of Communication Sciences have a high level of mobile phone ownership, daily use, and exposure to social media applications.

\section{Instrumentation}

A Likert-type scale was used to collect data in this study. The scale consisted of two main parts. In the first part, participants' personal information regarding their gender, school, department, duration of daily use, and ownership of mobile phone were asked through structured items. In the second part, "Bergen Facebook Addiction Scale" (BFAS), which was developed by Andreassen, Torsheim, Brunborg, and Pallesen (2014), was used to collect data about social media addiction of the participants. The fact that BFAS and parallel instruments were used in many international studies has allowed researchers to compare the results of the present study with the results of similar studies in other countries.

The validation process of the scale has taken place in several phases. First, all the 18 items in BFAS were translated into Turkish. Also, because the original scale was about Facebook addiction, the items were adapted to social media addiction. Secondly, language experts and social media specialists reviewed the translated version of the scale. Third, following several minor revisions, the draft was pilot-tested with 25 high school and 25 university students. A few revisions regarding the wording of the items were made. Finally, after the pilot-test, obtained data were analyzed and Cronbach Alpha reliability coefficient of the scale was calculated as .89 (it was found to be .90 in the actual study) so that the adapted scale was highly reliable.

Participants' mean scores were used to determine both how intensely each of the symptoms of addiction is experienced by individuals, and the level of social media addiction they experience. Having a total of 18 five-point items and the possible response for each item ranging from 1 to 5 , the minimum possible score for the whole scale was 18 whereas the maximum possible score was 90 . For the purpose of the study, the mean scores up to 20 (1.00-1.15 over 5 points) were used to describe a natural use, 21-39 (1.16- 2.21 over 5 points) mild addiction, 40-69 (2.22-3.87 over 5 points) moderate addiction, and $70-90$ (3.88-5.00 over 5 points) severe addiction. These were also the levels of addiction suggested in the original scale.

BFAS has been used to collect data in a number of countries and each study set the cut-off values for different levels of addiction. De Cook et al. (2014) in Belgium used the mean score of 2.00, Wang et al. (2015) in China used 3.00, and Jaffri (2015) in Bhutan used 4.00 as the critical score differentiating non-addicted and addicted users. On the other hand, Salem et al. (2016) in Egypt used the following score ranges to differentiate addiction levels: 0-20 (natural use), 20-39 (mild addiction), 40-69 (moderate addiction), and 70-90 (severe addiction).

The present study assumed that these four layers may serve as better indicators of social media addiction levels compared to a rigid dichotomous split as non-addicted and addicted. It was assumed that it is more reasonable to measuring the levels of addiction rather than simply labelling people as addicted versus non-addicted because sometimes there may be crucial differences among those categorized as addicted. 


\section{Procedures}

Data collection procedures were generally similar for both sub-samples. After obtaining necessary permissions from the selected schools, the researchers provided onsite instructors with elaborated information to administer the scale. While gathering data from high school students, the principal and a guidance teacher collaborated with the researchers. They administered the scale to their students as a part of guidance and counseling activities in the school. It took approximately four weeks in March 2017 to complete data collection in the high school. In the university setting, two professors with the help of their research assistants administered the scale to communication students during their compulsory courses. The administration of the scale to university students also took about four weeks during April 2017. All the students were informed about details of the study, participation was voluntary, and no rewards were given to the respondents for their attendance. More importantly, the high school teachers and the university professors have not taken any names from the students, nor they have shared any identity information with the researchers.

\section{Results}

\section{Gender}

Table 2 shows descriptive statistics, possible score ranges (minimum and maximum values) and t-test results with regard to gender.

Table 2. Results of t-test Regarding Gender Effects for All Participants

\begin{tabular}{|c|c|c|c|c|c|c|c|c|c|c|}
\hline \multirow[b]{2}{*}{ M } & \multicolumn{3}{|c|}{ Male $(n=355)$} & \multicolumn{4}{|c|}{ Female $(n=345)$} & \multicolumn{3}{|c|}{ General $(n=700)$} \\
\hline & SD & Min & Max & $M$ & SD & Min & Max & M & SD & $\mathrm{p}$ \\
\hline 2.35 & 0.73 & 1.00 & 5.00 & 2.54 & 0.80 & 1.00 & 5.00 & 2.44 & 0.76 & .001 \\
\hline
\end{tabular}

The mean addiction score of the total sample is at the moderate level $(M=2,44)$. This figure is neither too high nor too low but it deserves some attention. There was a meaningful difference between total addiction scores of the participants according to their genders. As seen in Table 2 , the mean score of female students $(M=2,54)$ is higher than the mean score of male students $(M=2,35)$. The difference between these two scores is significant $(p<.001)$. It is possible to conclude that female students are more addicted on social media than male students.

When considering the high school students, there are some gender differences on subdimensions of social media addiction. There are no significant differences between male and female students when we look at the dimensions of salience ( $M=2,50$ vs. $M=2,63 ; p=.163)$, tolerance $(M=2,67$ vs. $M=2,80 ; p=.214)$, relapse $(M=2,34$ vs. $M=2,51 ; p=.138)$, and conflict $(M=2,30$ vs. $M=2,42 ; p=.216)$. There is, however, a significant gender effect on mood modification ( $M=2,26$ vs. $M=2,61 ; p<.005)$ and withdrawal $(M=2,35$ vs. $M=2,66 ; p<.008)$ dimensions. Mean scores of female high school students regarding the dimensions of both mood modification $(M=2.61)$ and withdrawal $(M=2.66)$ were significantly higher than the mean scores of male students ( $M=2.26$ for mood modification; $M=2.35$ for withdrawal). Yet both male participants $(M=2,39)$ and female participants $(M=2,61)$ appear to have moderate addiction levels so that the overall addiction level of participants was moderate $(M=2,51)$. 
Findings for university students has some similarities as well as differences. There were no statistically significant differences between male and female participants in terms of salience $(M=2.48$ vs. $M=2.58 ; p=.335)$, tolerance $(M=2.68$ vs. $M=2.89 ; p=.095)$, relapse $(M=1.74$ vs. $M=1.94 ; p=.090)$, withdrawal $(M=2.60$ vs. $M=2.58 ; p=.899)$, and conflict $(M=2.01$ vs. $M=2.11$; $\mathrm{p}=.411)$. When the mean scores of mood modification with regard to gender are compared, however, the mean score of female students is statistically higher than the mean score of male students ( $M=2.15$ vs. $M=2.58 ; p<.001)$. In this case, it is possible to say that female students are more addicted in terms of mood modification so they experience more emotional changes in their social media interactions.

\section{School Type}

Table 3 presents the findings regarding the addiction levels of high school and university students. As seen in the table, the mean addiction score of high school students $(M=2.51)$ is higher than the mean addiction score of university students $(M=2.36)$. The difference between the mean score of high school students and the mean score of university students was statistically significant $(p<.013)$. Therefore, it can be said that high school students are more addicted to social media compared to university students.

Table 3. Results of t-test for Comparisons of High School and University Students

\begin{tabular}{|c|c|c|c|c|c|c|c|c|c|c|}
\hline \multicolumn{4}{|c|}{ University (n=303) } & \multicolumn{4}{|c|}{ High School $(n=397)$} & \multicolumn{3}{|c|}{ General $(n=700)$} \\
\hline$M$ & SD & Min & $\overrightarrow{\text { Max }}$ & $\mathrm{M}$ & SD & Min & $\operatorname{Max}$ & $M$ & SD & $\mathrm{p}$ \\
\hline 2.36 & 0.76 & 1.00 & 5.00 & 2.51 & 0.77 & 1.00 & 5.00 & 2.43 & 0.76 & .013 \\
\hline
\end{tabular}

As mentioned before, the high school sub-sample included two types of high schools as Anatolian High School and Science High School sections. Further analyses showed that there was no significant difference between high school types with regard to salience ( $M=2,55$ vs. $M=2,58$; $p=.707)$, tolerance $(M=2,79$ vs. $M=2,68 ; p=.311)$, mood modification $(M=2,44$ vs. $M=2,44$; $p=.989)$, relapse $(M=2,40$ vs. $M=2,46 ; p=.623)$, conflict $(M=2,35$ vs. $M=2,37 ; p=.875)$. There was a meaningful difference only in the withdrawal dimension ( $M=2.39$ vs. $M=2.64 ; p<.034)$. Thus, it is possible to say that Anatolian High School students who started to the school with relatively low entry scores and who were less successful in the process than Science High School students, who had better entry scores and higher academic performance during the courses, experience more difficulties to leave social media as they wanted.

Department-based differences were examined for university students. There was a statistically significant departmental effect regarding the withdrawal dimension only. According to the outcomes of the follow-up test, students in the Journalism Department had lower addiction scores than their counterparts both in the Department of Public Relations and Advertising $(p<.004)$ and the Department of Communication Design and Management $(p<.020)$. No significant differences were found in other dimensions of addiction.

\section{Duration of Daily Use}

As indicated before, approximately $85 \%$ of students use social media for more than 2 hours per day. Situation is almost the same for high school and university students. Therefore, the impact 
of frequent or longer use of social media on addictive behaviors were examined. The results of ANOVA are exhibited in Table 4. It is clearly seen in table that duration of use (amount of daily time) has a significant impact on social media addiction $(p<.000)$.

Table 4. ANOVA Results for Duration of Daily Use

\begin{tabular}{llllll}
\hline & Sum of Squares & $\mathrm{df}$ & Mean Square & $\mathrm{F}$ & $\mathrm{p}$ \\
\hline Between Groups & 92.982 & 3 & 30.994 & 66.197 & .000 \\
Within Groups & 325.870 & 696 & .468 & & \\
Total & 418.851 & 699 & & & \\
\hline
\end{tabular}

After ANOVA test, multiple comparisons were made for varying amount of daily time spent on social media (ranging from less than an hour to more than five hours per day). All comparisons produced significant results $(p<.001)$. The biggest difference was observed between the minimum amount of time to maximum amount of time (mean difference was 1,21/5,00). Therefore, it may be safe to say that when the time spent for the use of social media increases, the level of addiction also increases. Stated alternatively, the lesser the time, lower the addiction. Further analyses showed that more exposure to social media increases the level of addiction in all dimensions. This is due to the fact that the correlation coefficients among subdimensions of social media addiction are all positive and significant (see Table 5).

Table 5. Addiction Dimension Correlation Values

\begin{tabular}{lcccccc}
\hline & Salience & Tolerance & Mood & Relapse & Withdrawal & Conflict \\
\hline Salience & \multirow{2}{*}{1.000} & $.554^{* *}$ & $.287^{* *}$ & $.343^{* *}$ & $.348^{* *}$ & $.335^{* *}$ \\
Tolerance & & 1.000 & $.392^{* *}$ & $.492^{* *}$ & $.507^{* *}$ & $.468^{* *}$ \\
Mood & & & 1.000 & $.312^{* *}$ & $.308^{* *}$ & $.410^{* *}$ \\
Relapse & & & & 1.000 & $.431^{* *}$ & $.609^{* *}$ \\
Withdrawal & & & & & 1.000 & $.399^{* *}$ \\
Conflict & & & & & & 1.000 \\
\hline
\end{tabular}

Significant at .05, ** Significant at .01

The lowest correlation is between salience and mood modification ( $r=.287)$, while the highest correlation is between relapse and conflict $(r=.609)$. The correlations among dimensions of social media addiction are at medium or high levels. When separate calculations were made for high school and university students, the general situation was similar for both groups. The lowest correlation was between salience and mood modification ( $r=.231$ for high school and $r=.296$ for university students), while the highest correlation is between relapse and conflict ( $r=.600$ for high school students and $r=.595$ for university students).

\section{Discussion and Conclusions}

This study was conducted to identify and compare social media addiction levels of high school and university students. Demographic variables such as gender, school, department, duration of use, and dimensions of social media addiction (salience, tolerance, mood modification, relapse, withdrawal, conflict) are used as comparison variables. A total of 700 students participated in the study. The "Facebook Addiction Scale", which composed of 18 items and developed by 
Andreassen et al. at Bergen University, was adapted to social media addiction. The results of the study are discussed below.

Students participating in this study reported a moderate level of social media addiction. As of today, this level is not alarming but it may soon go up considering the incremental trend in use. This suggests that if the right steps are taken in advance, it will be possible in the future to reduce or overcome this type of addiction among students in Turkey. The studies, which are conducted in different countries based on Bergen Facebook Addiction Scale showed that while in the United States and West European countries students have a natural use or mild addiction level, in Eastern countries students have a moderate or severe addiction level. This might be considered normal because much of the technology was produced in Western (or developed) countries and these countries have already experienced and come over certain problems caused by the new technology. The Eastern (or developing) countries, on the other hand, take the technology from the West and follow their problems from one step behind. This difference can be considered in the context of the digital divide issue. From this point, Turkey may be accepted as a country which confronts the problem but does not know how to deal with it when it comes to social media addiction. While discussing and studying the addiction problem this should be taken into consideration.

When addiction levels of high school students and university students are compared, it is found that social media addiction level of the high school students is significantly higher than social media addiction level of the university students. It can be claimed that factors such as characteristics of age group, educational status, and intensified test anxiety (even stress and depression that high school students have to cope with) may be effective in this regard because preparation to university entrance exam is a painful work in Turkey. High school students, particularly those in the Anatolian High School, face anxiety or frustration due to the university entrance exam without knowing how to deal with it properly. They tend to have more online chats of other forms of interpersonal communications with their peers compared to university students who are free of such a pressure. This result is supported with the results of Zaffari's (2015) and Wang's (2015) studies revealing that that social media addiction is related to depression and anxiety of users; that is, as the levels of depression and anxiety of individuals increase, they become more inclined towards social media addiction. Therefore, it may be helpful for researchers to devote themselves to this issue in future studies in order to obtain more elaborate information.

Gender played a significant role in this respect. It appears that female students are more addicted on social media than male students. When considering high school students, there are gender differences on the dimensions of mood modification and withdrawal but other differences are not statistically significant. Findings for the university students have some similarities as well as differences. There were no significant differences between male and female university students in terms of salience, tolerance, relapse, withdrawal, and conflict. However, the gender difference for mood modification was significant, females being more addicted. Gender was found as an important factor affecting social media addiction by Steggink (2015) in the Netherlands and by Monacis et al. (2017) in Italy. On the other hand, there is no relationship between gender and social media addiction on the results of Wang et al. (2015) in China, Jafarkarimi et al. (2016) in Malaysia, and Blachnio et al. (2016) in Poland. This leads to a cross-cultural interpretation of the fact that gender has an impact on the use of social media in some countries. Thus, each culture should be studied in a deeper way within its own specific conditions in terms of gender. 
The results suggest that Anatolian High School students stay more on social media than Science High School students. Science High School students have a more demanding curriculum but they are elected based on their high aptitude scores while entering the school. Students who study in these high schools concentrate more on their courses and manage their time efficiently: This kind of behaviors may be effective in their low addiction scores compared to Anatolian High School students. Similarly, when we look at the departmental effects on social media addiction, it is observed that academic department in which students pursue an undergraduate degree has an impact on salience and withdrawal but not on other dimensions. Indeed, according to Zaffari et al. (2015) study on Pakistani students, there is a negative relationship between academic performance and Facebook addiction. It will be useful for researchers to focus on this issue in future studies so that more reliable results may be obtained.

Another important factor measuring the effect on social media addiction is duration of daily use. It has been found that both a strong majority of high school students $(84 \%)$ and university students (87\%) use social media for at least two hours a day. However, as the duration of social media use increases, the level of addiction (with all dimensions) increases. This situation can be explained with the increase in daily time spent on social media applications and the increase of the connection with the virtual life that the individual has established there. The addiction study of Jaffarkarimi et al. (2016) on students in Malaysia also shows that time of use is an important factor affecting addiction. In this sense, the results of the present study are consistent with others.

The comparison of the social media addiction level of students in Turkey with different countries will be helpful in seeing where Turkey stands on this issue. Although the data gathering instruments used in some of the studies are different from the one (BFAS) used in the present study, their core elements and corresponding items are similar in nature so that comparisons appear to be possible without serious complications. In a study conducted by Salem et al. (2016) in Egypt and Kuwait, participants' addiction levels were reported as 49.5 over 90 on average. In addition, the studies in Belgium and Germany show that addiction in these countries is not yet fully developed. According to Idubor's work in Nigeria, there is a high social media addiction among students in this country (Idubor, 2015). Finally, according to the study by Zhang et al. (2008) comparing the addiction level of young people in USA and China, $14 \%$ of students were heavily addicted to the Internet, $64 \%$ were slightly addicted, and $22 \%$ were not addicted. Among the U.S. respondents, $4 \%$ were heavily addicted to the Internet, $23 \%$ were slightly addicted, and $73 \%$ were not addicted. These figures show that social media addiction is a phenomenon that varies from culture to culture or country to country. This can be explained by variables such as countries' cultures, educational systems, social structures, practical opportunities, and the relationship with technology. Nevertheless, it would be useful to carry out research that compare cross-cultural social media addiction so that the causes of this situation can be better understood.

The present study is limited in certain ways. First, it was conducted in one private high school system and one college of communication in Western Turkey. It appears that the students in these schools have easy access to social media and a high level of mobile phone ownership. This may not be the case in the rest of Turkey. Considering that this situation might have affected the results, new studies should be conducted in a larger context that will better represent the overall situation in the country. Second, the data in the current study came from a sample of high school and university students. The level and characteristics of social media addiction may be different for adults; therefore, future studies should investigate the situation in adult populations. By doing so, we may have a chance to compare generational aspects of social media 
addiction. Finally, technology use may be influenced by the culture in which people grow up and act. Thus, cross-cultural studies may provide deeper understanding of social media addiction. Particularly, west-east comparisons may be useful based on the fact that there are differences between the levels of social media addiction in the western and eastern countries, which cannot be explained with penetration rates.

As a final remark, the use of social media is increasing at a very rapid pace in Turkey as the case in many other countries. This situation brings the risk of social media addiction, especially among young people. As the current study shows, this kind of addiction has concentrated particularly on the uses of high school and university students. In order to proceed further in a healthy manner, effective measures should be taken in social, political, cultural, and educational contexts. Relevant actions should focus particularly on improving legal regulations, educational opportunities, public awareness, psychological services, media consciousness, technological skills, parental guidance, and individual attentiveness.

\section{References}

American Psychiatric Association. (2013). Handbook of diagnostic and statistical manual of mental disorders (DSM-5). Washington, DC: The author.

Andreassen, S.C., Torsheim, T., Brunborg, G., Pallesen, S. (2014). Development of a Facebook addiction scale. Psychological Reports, 110(2), 501-517.

Blachnio, A., Przepiorka, A. \& Pantic, I. (2016). Association between Facebook addiction, selfesteem and life satisfaction: A cross sectional study. Computers in Human Behavior, 55, 701-705.

Cao, F., Su, L., Liu, T., \& Gao, X. (2007). The relationship between impulsivity and Internet addiction in a sample of Chinese adolescents. European Psychiatry, 22(7), 466-471.

Caplan, S.E. (2002). Problematic internet use and psychosocial well-being: Development of a theory based cognitive-behavioral measurement instrument. Computers in Human Behavior, 18(2), 553-575.

Colwell, J. \& Kato, M. (2003). Investigation of the relationship between social isolation, selfesteem, aggression and computer game play in Japanese adolescents. Asian Journal of Social Psychology, 6, 149-158.

Davies, T. \& Cranson, P. (2008). Youth work and social networking. Retrieved October 1, 2017 from http://www.nya.org.uk/wp-content/uploads/2014/06/Youth-Work-and-SocialNetworking-Final-Report-August-2008.pdf

De Cock, R., Vangeel, J., Klein, A., \& Meerkerk, G. (2014). Compulsive use of social networking sites in Belgium: Prevalence, profile, and the role of attitude toward work and school. Cyberpsychology, Behavior and Social Networking, 17(3), 166-171.

Griffiths, M.D. (2013). Social networking addiction: Emerging themes and issues. Journal of Addiction Research \& Therapy, 4(5). doi:10.4172/2155-6105.1000e118

Griffiths, M.D. \& Pontes, H.M. (2014). Internet addiction disorder and internet gaming disorder are not the same. Journal of Addiction Research \& Therapy, 5(4), doi:10.4172/2155$6105.1000 \mathrm{e} 124$. 
Huang, H. \& Leung, L. (2009). Instant messaging addiction among teenagers in China: Shyness, alienation and academic performance decrement. Cyberpsychological Behavior, 12(6), 675-679.

Idubor, I. (2015). Investigating social media usage and addiction levels among undergraduates in University of Ibadan, Nigeria. British Journal of Education, Society \& Behavioural Science, 7(4), 291-301.

Jaffarkarimi, H. et al. (2016). Facebook addiction among Malaysian students. International Journal of Information and Education Technology, 6(6), 465-469.

Jaffri, H. (2015). A study on Facebook addiction and its relationship with emotional experience and coping strategies. The International Journal of Indian Psychology, 2(4), 6-14.

Kerberg, C. S. (2005). Problem and pathological gambling among college athletes. Annals of Clinical Psychiatry, 17(4), 243-247.

Kim, K. et al. (2009). Internet overuse and excessive daytime sleepiness in adolescents. Psychiatry Clin Neurosci, 63(4), 455-462.

Koc, M. \& Gulyagci, S. (2013). Facebook addiction among Turkish college students: The role of psychological health, demographic, and usage characteristics. Cyberpsychol Behavior Social Network, 16(4), 279-284.

Kuss, D. \& Griffiths, M.D. (2011). Excessive online social networking: Can adolescents become addicted to Facebook? Education and Health, 29(4), 68-71.

Lin, M. P., Ko, H. C., \& Wu, J. Y. (2011). Prevalence and psychosocial risk factors associated with internet addiction in a nationally representative sample of college students in Taiwan. Cyberpsychology, Behavior, and Social Networking, 14, 741-746.

Monacis, L., de Palo, V., Griffiths, M. D., \& Sinatra, M. (2017). Social networking addiction, attachment style, and validation of the Italian version of the Bergen Social Media Addiction Scale. Journal of Behavioral Addictions, 6(2), 178-186.

Morahan-Martin, J. \& Schumacher, P. (2000). Incidents and correlates of pathological internet use among college students. Computers in Human Behavior, 16(1), 13-29.

Peter, J. \& Valkenburg, P. M. (2006a). Adolescents' exposure to sexually explicit online material and recreational attitudes toward sex. Communication Research, 56, 639-660.

Rosen L.D., Whaling, K., Carrier L.M., Cheever, N.A., \& Rokkum, J. (2013). The media and technology usage and attitudes scale: An empirical investigation. Computers in Human Behavior, 29(6), 2501-2511.

Salem, A. A., Almenaye, N.S., \& Andreassen, C.S. (2016). A psychometric evaluation of Bergen Facebook Addiction Scale (BFAS) of university students. International Journal of Psychology and Behavioral Sciences, 6(5), 199-205.

Shaffer, H.J. (1996) Understanding the means and objects of addiction: Technology, the Internet, and gambling. Journal of Gambling Studies, 12(4), 461-469.

Steggink, B. W. (2015). Facebook addiction: Where does it come from? A study based on the Bergen Facebook Addiction Scale (Unpublished master's thesis). University of Twente. The Netherlands.

Sussman, S., Liha, N., \& Griffiths, M. (2011). Prevalence of the addictions: A problem of the majority or the minority? Evaluation and the Health Professions, 34(1), 3-56. 
Tokunaga, R. S. (2011). Social networking site or social surveillance site? Understanding the use of interpersonal electronic surveillance in romantic relationships. Computers in Human Behavior, 27(2), 705-713.

Wang, C. W., Ho, R. T., Chan, C. L., \& Tse, S. (2015). Exploring personality characteristics of Chinese adolescents with Internet-related addictive behaviors: Trait differences for gaming addiction and social networking addiction. Addictive Behaviors, 42, 32-35.

Yen, C. Ko, C. Yen. (2007). The comorbid psychiatric symptoms of internet addiction: attention deficit and hyperactivity disorder (ADHD), depression, social phobia, and hostility. Journal of Adolesc Health, 41, 93-98.

Young, K. (1998). Internet addiction: The emergence of a new clinical disorder. CyberPsychology \& Behavior, 1(3), 237-244.

Zaffari, M. et al. (2015). Facebook addiction: Relation with depression, anxiety, loneliness and academic performance of Pakistani students. Sci. Int. (Lahore), 27(3), 2469-2475

Zhang, L., Amos, C., McDowell, C. (2008). A comparative study of Internet addiction between the United States and China. CyberPsychology and Behavior, 11(6), 727-729.

Correspondence: Ali Simsek, Professor, Department of Communication Design and Management, Faculty of Communication Sciences, Anadolu University, Yunus Emre Campus, Eskisehir, Turkey 